deceased athletes received no obituaries in the Soviet media, but the TASS correspondent in New York, Vladimir Kikilo, was able to confirm a number of the deaths. Given the uncertain provenance of the document, it would otherwise have been necessary to check its contents by a scan of the Soviet sporting press for the last

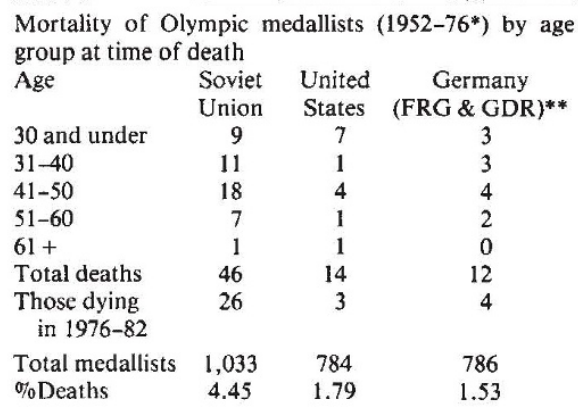

*The 1980 Moscow games are omitted from this survey due to the US boycott.

**Figures amalgamated because in 1952 East Germany did not compete and from 1956 to 1964 the two Germanies fielded a joint squad.

recorded appearance of the listed competitors.

The ban on the use of anabolic steroids for athletes makes it difficult to obtain statistically significant data on their longterm effects. Earlier this year, however, The American Journal of Sports Medicine listed no fewer than twelve steroid-related disorders, ranging from testicular atrophy in males and virilization in females to hepatic dysfunction, hypertension, changes in cholesterol composition, tumours, changes in connective tissue and psychosis.

There have also been suggestions that the Soviets may be administering to their athletes carnitine (involved in fatty acid metabolism), potassium orotate (an intermediary in the biosynthesis of pyrimidine nucleotides) and inosine (also involved in pyrimidine nucleotide synthesis and in the formation and breakdown of high-energy phosphates). Such compounds could serve both as performance enhancers and to counteract the short-term side effects of steroids. The long-term effects of high dosages are, however, unknown.

The mortality report on Soviet athletes - if accepted abroad - could serve as a grim warning to athletes and trainers in other countries who might feel tempted to resort to unauthorized aids. The status of the document, however, is still far from decided. Attempts by the "Smolosky" human rights group in the United States to bring it to the attention of the International Olympic Committee in Los Angeles proved unsuccessful.

Part of the explanation for that may have been the absence of a Soviet Olympic team, which in turn is held by many in Eastern Europe as well as the West to have been caused by Soviet reluctance to submit athletes to the sophisticated drug tests installed at Los Angeles.

\title{
Heavy-ion research
}

\section{European ambitions multiply}

GANIL, the new French accelerator for heavy ions at Caen in Normandy, is to close down for January to save electricity. GANIL must save 12 per cent as its contribution to last year's budget cuts, but director Claude Detraz is not perturbed. "We're stopping in January when electricity is most expensive", he says, "and anyway we're finding we've got plenty to do when the machine's off."'

In fact, Detraz is buoyant about the performance of his accelerator. He is even happy with his budget - which he believes to be quite sufficient to "sweep the field" in intermediate energy heavy-ion collisions. Detraz claims that GANIL is three years ahead of any other accelerator in performance and beam intensity and is making many interesting - and unexplained discoveries. What GANIL needs, he says, is not money but a team of committed theorists to help explain the effects being revealed.

The accelerator at Caen is the latest of a clutch of European heavy-ion machines (others are in West Germany and Britain) dedicated to the study of the gross properties of nuclei rather than the structure of nucleons and other particles, with which high-energy physics is now preoccupied.

Detraz says he is enticing French theorists to GANIL with discoveries such as that of isolated excited states of the nucleus 100 $\mathrm{MeV}$ above the ground state (where previously only a continuum of states was expected), the apparent failure of thermodynamic models of the excited nucleus and strong forward scattering (a kind of nuclear transparency) at total nuclear energies above $40 \mathrm{GeV}$. Other GANIL products (detectable because of high beam intensity) are exotic neutron-rich nuclei such as nitrogen-23, neon-29 and neon-30 as well as highly-charged positron-emitting atoms. "It's a gold-mine", says Detraz.

GANIL, however, is not alone in the mine. In Britain, working another seam, the Nuclear Structure Facility (NSF) may have taken a lead in high-spin nuclear physics, where nuclei are spun to such angular momenta that Coriolis and centrifugal forces are comparable with nuclear forces. NSF is planning a major extension of its Van de Graaff accelerator, the largest in existence, by means of a superconducting linac.

In West Germany also, the heavy-ion laboratory (GSI) near Darmstadt is basking in the discovery of elements 108 and 109 , and has won outline approval for a substantial extension. And Italy is building an advanced superconducting cyclotron in Milan, although the project was set back recently by the untimely death of its designer.

GANIL itself uses a pair of separatedsector cyclotrons to accelerate a wide range of nuclei from $100 \mathrm{MeV}$ per nucleon (for light ions) up to $10 \mathrm{MeV}$ per nucleon (for the heaviest). In low-energy nuclear accelerators (such as NSF), nuclei approach at velocities that are small compared with the internal velocities of their nucleons, so that internal structures are rearranged before collisions. At much higher energies (such as the $\mathrm{GeV}$ per nucleon available at Berkeley, California), on the other hand, collision velocity is much faster than the internal motions, and nuclei collide like bags of stationary separate particles. But at GANIL - the intermediate region - the velocities are roughly equal, so that simple approximations do not apply. Hence perhaps, the lack of enthusiasm of theorists, whom Detraz claims are nevertheless being won round by the challenge of GANIL.

NSF (at Daresbury, Cheshire) is making its mark because of its stable and turnable particle beams (a consequence of eletrostatic acceleration), the range of nuclei which can be accelerated ("virtually the whole periodic table" according to a Daresbury experimenter), and a superb detector of gamma-emission from high spin states which can discriminate against Compton-scattered radiation.

Daresbury now plans to abandon the original target of a $30 \mathrm{MV}$ terminal voltage, to stay with the present $20 \mathrm{MV}$ but to put all its effort into developing a superconducting linac stage after the Van de Graaff. This would give NSF the capability of colliding uranium with uranium. The proposal has not yet gone to the nuclear physics board of the Science and Engineering Research Council that would fund it, but the extension could be built segment by segment without increasing the budget, they say in Cheshire.

Meanwhile, GSI Darmstadt plans to add a synchrotron and a storage ring to its present UNILAC linac (which is capable of colliding nuclei at around $20 \mathrm{MeV}$ per nucleon). The additions would bring GSI up to $1.3 \mathrm{GeV}$ per nucleon at high intensity, making possible a search for new states of condensed nuclear matter. The GSI additions are all but finally approved, a federal government spokesman in Bonn said last week, with a decision due "before the end of the year'".

With work in this field also at CERN (the European Organisation for Nuclear Physics near Geneva) and progress in Italy, the chauvinistic cry is getting louder in Europe that the old continent has taken "an unquestionable world lead" in nuclear matter studies. That, of course, was what the particle physicists were saying until President Reagan gave the go-ahead to the super-conducting super-collider now being built as an adjunct to the Stanford Linear Accelerator Center and which may steal a march on CERN's new large electronpositron storage ring. Robert Walgate 